this number even further. Such on-demand generation of entanglement was also reported recently ${ }^{5}$ using a different chip implementation from that of Kurpiers and colleagues.

The direct-transfer strategy has also been carried out using optical photons between distant atoms or ions ${ }^{6}$. In that case, reaching high efficiencies of photon emission and capture remained a challenge. But another way to overcome the prohibitive scaling of quantum networks is to turn a probabilistic method into a deterministic one.

Under a probabilistic protocol, each attempt to produce entanglement has a low chance of success. However, if enough attempts are realized in a given length of time, the generation of entanglement can be ensured in this time frame. Such an approach can therefore provide deterministic entanglement at a predetermined time. But there is a crucial requirement for achieving this goal: entanglement must be produced more quickly than it is lost, or else the generated entanglement could be gone before it has been delivered.

In 2015, a trapped-ion experiment succeeded in breaking this threshold ${ }^{7}$. Humphreys and colleagues have now achieved the same feat using a solid-state system. In their work, the two quantum nodes were single defects, known as nitrogen-vacancy centres, in diamond (Fig. 1b). The authors placed diamonds in cooling devices that were separated by a distance of 2 metres. They then generated entanglement between the spins (magnetic moments) of the nitrogen-vacancy centres by adopting a 'heralded' technique that has been used for other platforms and has enabled rudimentary versions of quantumrepeater segments ${ }^{8,9}$.

Humphreys et al. prepared the two nodes so that they had an identical spin state. For each system, the authors used laser pulses to generate entanglement between the spin of the nitrogen-vacancy centre and the presence or absence of an optical photon emitted into a fibre. The fibres from both systems were connected, and the detection of a single photon midway between the nodes projected the two spins into an entangled state. This is because there was no way of knowing, even in principle, from which node the photon was emitted. This single-photon scheme was one of two key ingredients of the authors' work: it enabled a much higher rate of entanglement production than could be achieved in a previous study ${ }^{10}$ using nitrogen-vacancy centres that relied on a two-photon process.

The second key ingredient was a dramatic extension in the lifetime of the stored entanglement to a period of hundreds of milliseconds. The authors achieved this by protecting the stored state after it was produced. Overall, the combination of the two ingredients enabled entanglement to be generated almost ten times faster than it was lost. Thanks to this achievement, Humphreys and colleagues demonstrated a deterministic delivery of entanglement roughly every 100 milliseconds.

The long-awaited advances of these two research groups demonstrate that the prospect of realizing functional quantum networks - either on the local scale between superconducting modules or on a larger scale between communication nodes that are connected by optical fibres - is getting closer to reality (see Nature 554, 289-292; 2018). The numbers still need to be improved; in particular, the rate at which long-distance entanglement can be delivered remains too low for practical applications. However, an increase by a factor of about 100 should be achievable in the near future.

To demonstrate large-scale and long-haul quantum networks, a combination of techniques and tools will be necessary. Among other methods, complementary approaches that rely on ensemble-based quantum memories are being developed at a fast pace ${ }^{11}$. When combined with massive multiplexing in time, frequency or space - a process that has been necessary for the development of the Internet - these methods should be able to provide entanglement at a high rate.

Another important goal will be the realization of an efficient quantum converter that links microwave photons to optical photons ${ }^{12}$.
Such a device should enable the platforms used in these two studies, which have different capabilities, to be connected. Putting these blocks together will be a tremendous challenge for science and engineering, but it promises to lead to versatile networks in which quantum processors are interconnected through a quantum-communication web.

Julien Laurat is at the Laboratoire Kastler Brossel (Sorbonne Université, CNRS, ENS, Collège de France), Sorbonne Université, Campus Pierre et Marie Curie, 75005 Paris, France.

e-mail:julien.laurat@sorbonne-universite.fr

1. Kurpiers, P. et al. Nature 558, 264-267 (2018).

2. Humphreys, P. C. et al. Nature 558, 268-273 (2018)

3. Kimble, H. J. Nature 453, 1023-1030 (2008).

4. Cirac, J. I., Zoller, P., Kimble, H. J. \& Mabuchi, H. Phys. Rev. Lett. 78, 3221-3224 (1997).

5. Axline, C. J. et al. Nature Phys. https://doi. org/10.1038/s41567-018-0115-y (2018).

6. Reiserer, A. \& Rempe, G. Rev. Mod. Phys. 87 1379-1418 (2015).

7. Hucul, D. et al. Nature Phys. 11, 37-42 (2015).

8. Duan, L.-M., Lukin, M. D., Cirac, J. I. \& Zoller, P. Nature 414, 413-418 (2001).

9. Chou, C.-W. et al. Science 316, 1316-1320 (2007).

10.Pfaff, W. et al. Science 345, 532-535 (2014).

11. Maring, N. et al. Nature 551, 485-488 (2017).

12.Andrews, R. W. et al. Nature Phys. 10,321-326 (2014).

\title{
IMMUNOLOGY
}

\section{Tumour tamed by transfer of one T cell}

The T cells of the immune system can be engineered to target a tumour, but why some people respond better than others to such therapy is unclear. One patient's striking response to treatment now offers some clues. SEE LETTER P.307

\section{MARCELA V. MAUS}

$\mathrm{T}$ he use of genetically engineered immune cells to target tumours is one of the most exciting current developments in cancer treatment. In this approach, $\mathrm{T}$ cells are taken from a patient and modified in vitro by inserting an engineered version of a gene that encodes a receptor protein. The receptor, known as a chimaeric antigen receptors (CAR), directs the engineered cell, called a CAR T cell, to the patient's tumour when the cell is transferred back into the body. This therapy can be highly effective for tumours that express the protein CD19, such as B-cell acute leukaemias ${ }^{1,2}$ and large-cell lymphomas ${ }^{3,4}$. However, some people do not respond to CAR T cells, and efforts to optimize this therapy are ongoing. On page 307, Fraietta et al. ${ }^{5}$ report the fortuitous identification of a gene that positively affected one person's response to treatment with CAR T cells.
Therapies involving engineered immune cells use viral vectors based on retroviruses or lentiviruses to insert a DNA sequence, such as one encoding a CAR, into a person's T cells. However, given that there is no control over where the sequence inserts into the genome, it is possible that the engineered gene could insert at a location that disrupts another, important gene. In the early 2000 s, a clinical trial $^{6}$ enrolled people with immunodeficiencies arising from the lack of a functional copy of a particular immune gene. The trial used viral vectors to insert a wild-type copy of this gene into their stem cells. Unfortunately, however, several people developed uncontrolled T-cell proliferation that evolved into T-cell leukaemia. This event was linked ${ }^{7}$ to the gene inserting within the sequence of the LMO2 gene, disrupting the normal regulation of $L M O 2$.

The pattern of genomic integration sites for various viral vectors has been found to be specific for a given combination of vector 


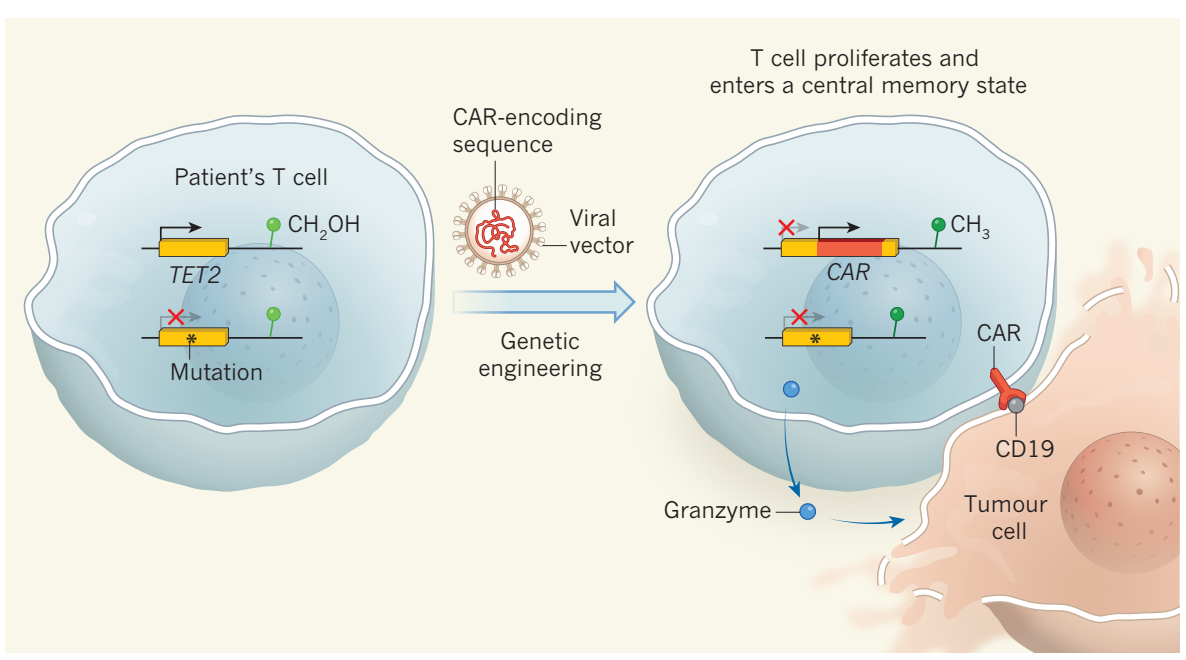

Figure 1 | Tumour targeting by CAR T cells. If a patient's T cells are engineered to express a version of an immune-cell receptor called a CAR, the cells can target tumour cells that express a specific protein, such as CD19. However, not everyone responds to this treatment. Fraietta et al. ${ }^{5}$ report that one patient's response to CAR T-cell treatment has revealed a gene that can affect therapy success. The patient had a mutation in one of their copies of the TET2 gene. TET2 encodes an enzyme that converts methyl $\left(\mathrm{CH}_{3}\right)$ groups attached to DNA into hydroxymethyl $\left(\mathrm{CH}_{2} \mathrm{OH}\right)$ groups. This type of change is known as an epigenetic modification. When a CAR-encoding sequence was introduced into the patient's T cells using a viral vector, in one cell the CAR sequence inserted into the patient's non-mutated copy of TET2 and disrupted the gene, thereby generating a cell that lacked any functional copies of TET2. The clonal descendants of this cell eradicated the patient's tumour. The lack of TET2 altered the cell's profile of epigenetic modifications, which can affect gene expression. This TET2 deficiency was associated with an increase in the expression of tumour-killing factors such as the enzyme granzyme, as well as entry into a cellular state called the central memory state, which stops the cells from entering a dysfunctional mode called exhaustion.

and cell type ${ }^{8}$. In a study of people who had $\mathrm{T}$ cells modified using retroviral vectors, the integration events were not implicated as the cause of any cancers ${ }^{9}$. Lentiviral vectors integrate randomly into the genome but tend to preferentially locate at sites of transcriptionally active genes ${ }^{10}$. Although random integration is generally thought to be safe, any disruption of the genome nevertheless confers a risk of unwanted consequences.

The effectiveness of treatments involving CAR T cells has been linked to the persistence and proliferation of the CAR T cells in the person's body, and this can be affected by factors including the disease subtype, the molecular design of the CAR used, and even the manufacturing process ${ }^{1}$. Fraietta et al. report the unusual response of a person in a clinical trial whose CAR T cells targeted a CD19-expressing tumour called chronic lymphocytic leukaemia. In this case, disruption of the gene into which the CAR sequence had been inserted had a direct and beneficial effect on the clinical outcome.

The patient began to show a noticeable response to treatment two months after receiving a second dose of CAR T cells. Tumour regression normally occurs within a month if treatment is successful, so the authors investigated the reason for the delay in this case. Crucially, they analysed the nature of the CAR T cells at peak concentrations in the blood during tumour regression. Fraietta and colleagues made the surprising observation that these CAR $\mathrm{T}$ cells consisted almost exclusively of a clonal population descended from a single cell.

This single cell's progeny divided over time until the cellular descendants reached a tipping point that eliminated the entire tumour. It is remarkable that the minimally effective and curative dose of this form of immunotherapy can be the introduction of just one cell. This raised the question of why introducing the CAR sequence to this specific $\mathrm{T}$ cell caused such an effective antitumour response.

In this clonal population of $\mathrm{T}$ cells, the CAR sequence had inserted into a copy of the TET2 gene, preventing the gene from encoding a functional protein. The patient's other copy of TET2 had a mutation, so insertion of the CAR sequence generated $\mathrm{T}$ cells that lacked TET2 protein. TET2 is an enzyme, also called methylcytosine dioxygenase, that catalyses a hydroxylation reaction that alters methyl groups attached to DNA (Fig. 1). Such modifications of DNA or its associated proteins are known as epigenetic modifications, and they can affect gene expression in some cases. When Fraietta and colleagues compared the patient's $\mathrm{T}$ cells that lacked the CAR insertion with those into which the CAR had been inserted, the overall epigenetic profile was similar. However, differences in the structure of the DNAprotein complex called chromatin were observed in genes involved in T-cell function, including CD28, ICOS and the gene that encodes interferon- $\gamma$.

TET2 mutations have previously been associated with clonal blood-cell alterations linked to a risk of disease or blood cancers (a phenomenon known as clonal haematopoiesis) ${ }^{11}$. However, the patient's $\mathrm{T}$ cells that lacked TET2 did not give rise to either aberrant T-cell proliferation or cancer. After tumour elimination, the number of CAR $T$ cells decreased appropriately, replicating the normal pattern for a T-cell population (increasing in response to its target and declining after target elimination).

The authors used genetic engineering to remove TET2 in human T cells in vitro. Analysis of these cells revealed a connection between the absence of TET2 and the promotion and maintenance of $T$ cells in a cellular state known as a central memory state. This state helps to prevent the cells from entering a dysfunctional mode called exhaustion, which is linked to ineffective tumour targeting by T cells. The absence of TET 2 was also linked to an increase in long-term T-cell memory.

Fraietta et al. observed that human T cells lacking TET2 made fewer immune signalling molecules called cytokines than did cells that had TET2. Disruption of TET2 was also linked to an increase in the level of the enzymes perforin and granzyme, which are components of the tumour-killing machinery of T cells. These roles of TET2 in T-cell function and memory were previously unknown.

These remarkable findings might suggest that targeting TET2 in human T cells through drug-mediated inhibition or gene-editing techniques could increase the effectiveness of CAR T-cell treatment for other patients. If so, perhaps the dose of CAR T cells needed might be only a few cells, rather than the usual 50 million to 500 million cells. This would shorten the waiting time for CAR T cells and lower the substantial manufacturing costs. However, given the known associations of TET2 mutations with certain disease states, this approach might run the risk of generating a malignancy.

Enhancing CAR T-cell function is an area of active research, and other options to achieve this goal have been proposed. Inserting a CAR sequence at the genomic location where the natural version of the gene resides enhances the activity and persistence of CAR T cells in a mouse model ${ }^{12}$. Other groups have reported progress ${ }^{13}$ in making CAR T cells resistant to inhibitory checkpoint-signalling pathways that hinder T-cell function.

Will one of the many possible approaches be preferable to the others? Unfortunately, animal studies are not always predictive of results in humans, so clinical trials are the only way to answer this definitively. The good news is that it seems likely that many of these approaches will enhance efficacy and safety, so there is hope that the use of CAR T cells to treat cancer will become even more successful in the years to come.

Marcela V. Maus is in the Department of Medicine, Division of Hematology and Oncology, Harvard Medical School, and the Massachusetts General Hospital Cancer 
Center, Boston, Massachusetts 02114, USA. e-mail:mvmaus@mgh.harvard.edu

1. Maus, M. V. \& June, C. H. Clin. Cancer Res. 22, 1875-1884 (2016)

2. Park, J. H. et al. N. Engl. J. Med. 378, 449-459 (2018).

3. Neelapu, S. S. et al. N. Engl. J. Med. 377, 2531-2544 (2017)
4. Schuster, S. J. et al. N. Engl. J. Med. 377, 2545-2554 (2017).

5. Fraietta, J. A. et al. Nature 558, 307-312 (2018)

6. Hacein-Bey-Abina, S. et al. N. Engl. J. Med. 346, 1185-1193 (2002).

7. Hacein-Bey-Abina, S. et al. Science 302, 415-419 (2003).

8. Biasco, L. et al. EMBO Mol. Med. 3, 89-101 (2011).
9. Scholler, J. et al. Sci. Transl. Med. 4, 132ra53 (2012) 10.Schröder, A. R. W. et al. Cell 110, 521-529 (2002).

11.Buscarlet, M. et al. Blood 130, 753-762 (2017).

12. Eyquem, J. et al. Nature 543, 113-117 (2017).

13 Ren, J. et al. Clin. Cancer Res. 23, 2255-2266

(2016).

This article was published online on 30 May 2018.

\section{Remote wiggling helps cold enzymes work}

\section{Mutations introduced far from the active site of an enzyme can cause local unfolding that increases enzyme activity. This finding suggests how organisms that live in the cold can speed up biochemical reactions. SEE LETTER P.324}

\section{ASHOK A. DENIZ}

$\mathrm{T}$ The biochemical sciences have tended to focus on processes that take place at 'physiological' temperatures of around $37^{\circ} \mathrm{C}$. But much of Earth's surface is covered with ocean, ice or snow, and is replete with organisms that function at much lower temperatures. Life in these environments requires suitable biological adaptations, for example in the enzymes that maintain the chemical environment of the cell. On page 324, Saavedra et al. ${ }^{1}$ shed light on a biophysical mechanism for such low-temperature adaptation that operates at the molecular level. Their results show strikingly that protein modifications distant from an enzyme's active site can modulate localized unfolding of the enzyme effectively, wiggling of parts of the enzyme's structure - that can control several facets of enzyme-reaction mechanisms.

Physical chemists have long known that the rate of chemical reactions depends on temperature, and that reaction rates generally decrease as temperatures drop. This temperature dependence also applies to enzyme-catalysed reactions, raising the intriguing question of how psychrophilic organisms (which live at low temperatures) can maintain their repertoire of enzyme-mediated functions. Related enzymes in psychrophilic organisms and in mesophilic organisms (which live at physiological temperatures) have similar activities - that is, the reactions they catalyse occur at similar rates ${ }^{2}$. For this to occur, the functional parameters of the cold-adapted enzymes must have been tuned to compensate for the lower temperatures.

A clue to how this tuning could occur came from previous observations ${ }^{3}$ that psychrophilic enzymes tend to have more surface glycine mutations (in which an amino-acid residue on the protein's surface is replaced by a glycine residue) far from the active site than do similar enzymes in mesophilic organisms. However, the mechanistic details of this phenomenon were poorly understood. Saavedra et al. used an enzyme called adenylate kinase to test the mechanism by which such glycine mutations act from a distance to alter enzyme function.

Adenylate kinase catalyses reactions that help to maintain a balance of adenosine phosphates (molecules that act as the energy currency of cells). The authors chose this mesophilic enzyme because it has been used extensively as a model system for investigating enzyme biophysics, biochemistry and folding, including by researchers from the same laboratory as Saavedra and co-workers.

In the present work, the authors tested the previously discussed idea ${ }^{2}$ that tuning of entropy - a measure of disorder - is a major driving force in the adaptation of enzymes to low temperatures. They sought to probe the effect of surface glycine mutations, at locations far from the active site, that might change the 'wiggling' (an entropic effect) of the protein without changing its overall folded structure.

There are three domains in adenylate kinase: the CORE domain, which contains much of the active site, and LID and AMPbd, each of which contains part of the active site. The authors studied glycine mutations in both the LID and the AMPbd domains by using a combination of biophysical and structural techniques. These studies included measuring the stability of the enzyme variants and their binding affinity to a mimic of the enzyme's substrate, and a more detailed characterization of the protein states and structural fluctuations by using nuclear magnetic resonance spectroscopy.

Taken together, Saavedra and colleagues' results demonstrate that adenylate kinase exists in at least three different states, and that the mutations change the relative occupancy (stability) of these states. Compared with the

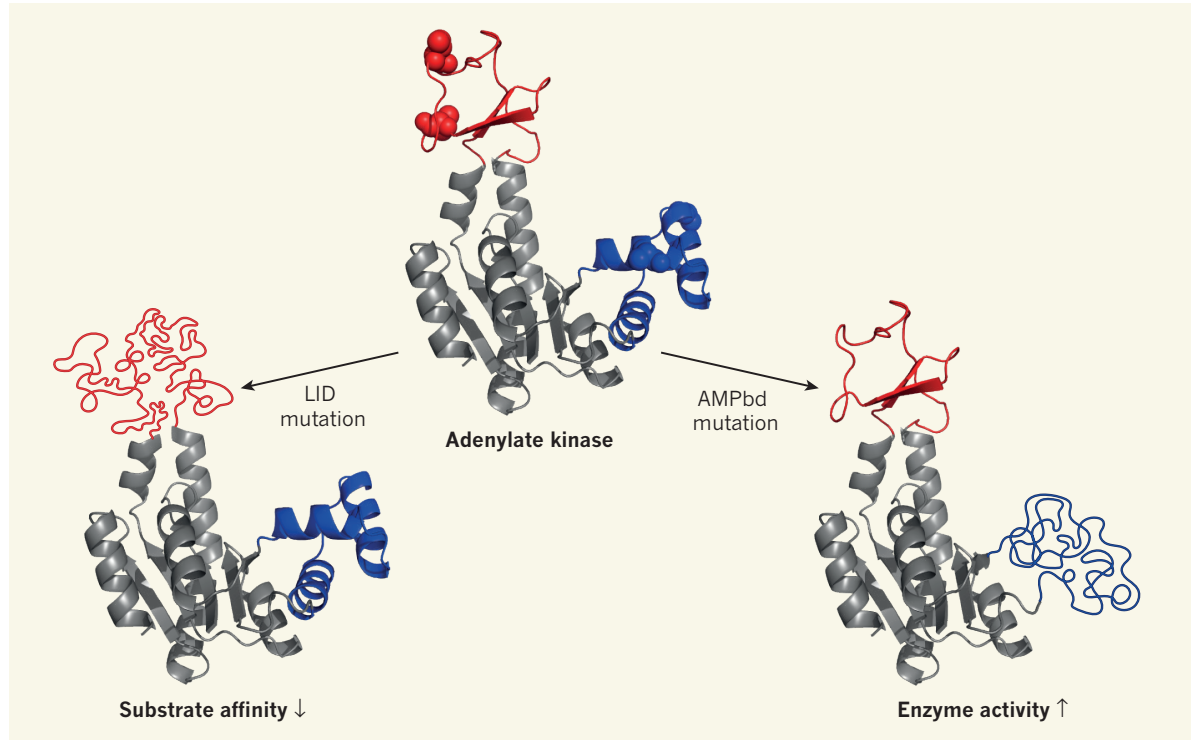

Figure 1 | Entropic tuning of enzyme function allows cold adaptation. Saavedra $\mathrm{et} \mathrm{al.}{ }^{1}$ made mutants of the enzyme adenylate kinase, replacing non-glycine amino-acid residues with glycine residues at the surface of either the LID domain (red) or the AMPbd domain (blue). Both types of mutation caused local unfolding of the enzyme, increasing its disorder (entropy), and altered the enzyme's functional behaviour, despite being distant from the active site. The LID mutations decreased the affinity of adenylate kinase for its substrates, whereas the AMPbd mutations increased the enzyme activity. Such entropic tuning of function might be an evolutionary mechanism that allows enzymes to cope with low temperatures, which usually slow enzymatic reactions. 